\title{
Existenzsicherung unter Risikobedingungen - Sozialwissenschaftliche Analyseansätze zum Umgang mit Krisen, Konflikten und Katastrophen
}

\section{Fred Krüger, Erlangen, Elísio Macamo, Bayreuth}

\section{Zur Anatomie des Risikobegriffs}

Risiko ist im Kontext der Entwicklungsforschung bisher oft als eine negative Rahmenbedingung für Alltagshandeln und Existenzsicherung interpretiert worden. Die entwicklungspolitische Implikation bestand folglich darin, möglichst risikolose, in diesem Sinne also sichere Lebensbedingungen zu schaffen. Es galt, eine riskante soziale oder natürliche Umwelt soweit zu entschärfen, dass die betroffenen Menschen in einem fortan weitgehend gefahrlosen Lebensumfeld ein nachhaltig gesichertes Auskommen erzielen würden. Im Unterschied dazu betonen einige jüngere sozialwissenschaftliche Ansätze die Bedeutung von Risiko als Vorbedingung für wirksame Überlebenssicherung. Indem Individuen oder Gruppen Risiken als Eintrittswahrscheinlichkeiten möglicher Handlungsergebnisse kalkulieren und sie damit von Unwägbarkeiten abgrenzen, entstehen überhaupt erst Bedingungen für eine erfolgreiche Lebenshaltung (vgl. JoHnston et al. 2000: 717). Risiko wird hier also geradezu zur Voraussetzung für eine effiziente Reduzierung der gesellschaftlichen Verwundbarkeit erklärt. Trotz dieser offensichtlichen Dissonanz der beiden Risikokonzeptionen ergeben sich aus der Kombination beider Ansätze wertvolle Hinweise zur Erklärung von Verwundbarkeit und Existenzgefährdung.

Ansätze aus der Anthropologie, Ethnologie und Soziologie sehen Risiko als Ergebnis gesellschaftlicher Perzeptionen, Entscheidungen und Handlungen und somit als kollektives Konstrukt (z.B. Douglas \& WiLDAVSKY 1982; Douglas 1986, 1992; Luhmann 1991; Yates 1992; SPITTLER 1994, 1996; vgl. auch eine Stellungnahme aus geographischer Sicht bei PoHL 1998). Gefahr steht bei diesen Konzeptionen vor Risiko. Eine soziale Gruppe, die einer Gefahr ausgesetzt ist und diese weder wahrnimmt noch interpretiert, geschweige denn auf der Grundlage dieser Interpretation angemessen reagiert, ist hochgradig verwundbar. Gesellschaften, denen es gelingt, Gefahren zu erkennen und einzuschätzen, sie also kalkulierbar zu machen, können gezielt Handlungsmuster der Lebensabsicherung entwickeln und ihre Verwundbarkeit reduzieren. Risiko ist hier also untrennbar mit Wahrnehmungen, Interpretationen und Handlungen verbunden; den Betroffenen wird eine zentrale und aktive Rolle im System von Gefahr/ Gefährdung und Existenzsicherung zugewiesen.
In einigen geographischen Ansätzen wird Risiko weniger als Ergebnis diskursiver Prozesse oder von Perzeptions- und Handlungsmustern, sondern vielmehr als Funktion von (Natur-)Gefahren und Verwundbarkeit beschrieben. BlaIKIE et al. (1994) betonen, dass Risiko dann nicht existiert, wenn es zwar ein Gefahrenereignis (hazard event, z.B. einen Vulkanausbruch oder eine Dürre) gibt, die Anfälligkeit der betreffenden Gesellschaft jedoch gleich Null ist. Ebenso sei eine Gesellschaft dann keinem Risiko ausgesetzt, wenn sie zwar verwundbar sei, es aber keine Gefahrenereignisse gäbe. Die einfache Formel «Risiko = Gefahrenereignis + Verwundbarkeit» ist Kern eines Modells, das die Lebenssituation von Menschen durch zwei gegeneinander wirkende Kräfte determiniert sieht: Auf der einen Seite stehen Prozesse, die zur Erhöhung gesellschaftlicher Verwundbarkeit führen, auf der anderen Seite wirkt der (potentielle) Eintritt eines Schadensereignisses. Je höher der Druck ist, den diese Klammer auf die betroffenen Menschen ausübt, umso größer ist das Risiko einer Krise oder Katastrophe. HewitT (1997) beschreibt vier Faktorenkomplexe, welche die Risikobedingungen menschlichen Daseins bestimmen: Erstens Gefahrenereignisse, zweitens Verwundbarkeit und Anpassungsfähigkeit, drittens mittelbare strukturelle Bedingungen der sozialen und natürlichen Umwelt (also Habitatsmerkmale) sowie viertens Bewältigungsstrategien und Interventionsmaßnahmen. Risiken erhalten damit eine für die betroffenen Gemeinschaften oder Individuen und einen bestimmten Ort ganz spezifische Qualität. Das Risiko, von einer Überschwemmung heimgesucht zu werden und das gesamte Hab und Gut zu verlieren, ist für Bewohner einer Flussaue generell größer als für Menschen in höher gelegenen Gebieten. Aber in der Nähe des Flusses sind außerdem jene Personen besonders gefährdet, denen die Möglichkeit fehlt, sich standfeste Häuser zu bauen oder die keine Warnhinweise erhalten, die es ihnen erlauben, sich und ihre Habe noch rechtzeitig in Sicherheit zu bringen. Vielleicht haben die am Flussufer Lebenden gar keine andere Wahl, als genau dort zu wohnen - sei es, weil sie als Fischer auf die Nähe zum Wasser angewiesen sind oder weil ihnen als Angehörige einer marginalisierten Gruppe der Zugang zu überschwemmungssicheren Siedlungsflächen versagt wird. Die Geographie des Orts und des sozialen Settings ist also integraler Bestandteil des Risikos (Hewitr 1997: 12).

Konkreter, aber wohl auch vereinfachender sind Definitionen, die anhand messbarer Indikatoren eine Risi- 


\begin{tabular}{|l|l|}
\hline \multicolumn{2}{|c|}{ Risikokonzeptionen } \\
\hline \multicolumn{2}{|c|}{ Risiko als Ergebnis einer Funktion von... }
\end{tabular}

Tab. 1: Risikokonzeptionen

Notions of risk

Conceptions du risque

koklassifikation erlauben (sollen) und damit politischen Entscheidungsträgern, die Vorsorgemaßnahmen einzuleiten bzw. dafür einen rechtlichen Rahmen zu schaffen haben, einen Handlungsrahmen bieten. So hat z.B. der Wissenschaftliche Beirat der Bundesregierung Globale Umweltveränderungen (WBGU) in seinem Jahresgutachten 1998 globale Umweltrisiken als Funktion von Eintrittswahrscheinlichkeit und Schadensausmaß definiert. Daraus werden sechs Risikotypen abgeleitet, die einer jeweils spezifischen politischen Behandlung bedürfen (WissensCHAFTLICHER Beirat der Bundesregierung Globale UmweltVERÄNDERUNGEN (WBGU) 1999). Der Typ «Damokles» etwa besitzt bei sehr geringer Eintrittswahrscheinlichkeit, die zudem sehr gut abgeschätzt werden kann, ein extrem hohes Schadenspotential, welches aber wiederum gut kalkulierbar ist. Dies trifft z.B. für Kernenergieanlagen oder Meteoriteneinschläge zu. Zum Umgang mit diesem Risiko werden drei Strategien empfohlen: Reduzierung des Katastrophenpotentials mittels Forschung und technischer Maßnahmen, Förderung der Robustheit gegenüber Überraschungen, indem institutionelle und organisatorische Strukturen gestärkt werden, und Aufbau eines effizienten Katastrophenmanagements als Nachsorgemaßnahme. Im Kontext der Überlebenssicherung sind vor allem die Typen «Zyklop» (z.B. Vulkanausbrüche, Überschwemmungen, AIDS) und «Kassandra» (z.B. Destabilisierung terrestrischer Ökosysteme) relevant, da einige Strategieempfehlungen unmittelbare Auswirkungen auf das Alltagshandeln der (potentiell) Betroffenen hätten, wenn sie umgesetzt würden. Angemahnt wird hier nämlich u.a. die Stärkung individueller Handlungskapazitäten im Sinne von empowerment oder Bildung.

Prinzipiell gehen alle hier vorgestellten Konzeptionen von der Annahme aus, dass es sich bei einer Risikosi- tuation um das Ergebnis einer Funktion von «Gefahr $+\mathrm{x} »$ handelt (Tab. 1). Der entscheidende Unterschied der Ansätze besteht in der Natur des «X». Es kann von Bedeutungszuschreibungen über die Wirksamkeit von Bewältigungsstrategien bis zu berechneten Häufigkeitsangaben und dem bezifferten Verlust von Sachwerten oder Menschenleben verschiedenste Werte annehmen. Das breite Wertespektrum führt folglich $\mathrm{zu}$ divergierenden entwicklungspolitischen Zielsetzungen. Grundsätzlich geht es darum, dafür zu sorgen, dass Risikosituationen beherrschbar bleiben. Drohen sie außer Kontrolle zu geraten, dann gerät - so argumentieren wir - ein soziales System in eine Krise; ist der Kontrollverlust eingetreten, sprechen wir von einer Katastrophe. Anhand von zwei Ansätzen der akteursorientierten Entwicklungsforschung, die zum einen aus geographischer, zum anderen aus soziologischer Sicht Probleme der alltäglichen Existenzsicherung auf der Ebene des Haushalts oder einer lokalen Gemeinschaft zu analysieren versuchen, legen wir unsere Überlegungen im folgenden dar.

\section{Krisenbewältigung aus Sicht der Verwundbarkeits- und livelihood-Modelle}

Seit einigen Jahren sind Analyseansätze der Entwicklungsforschung ins Blickfeld gerückt, die versuchen, die Existenzsicherung marginalisierter oder einkommensschwacher Bevölkerungsgruppen aus einer ganzheitlichen Perspektive zu beschreiben. Dabei hat vor allem der sustainable livelihoods approach Bedeutung erlangt. Er wurde im Wesentlichen von britischen Organisationen der Entwicklungszusammenarbeit (u.a. Department for International Development (DFID), Oxfam, Care) entworfen (vgl. CARNEY et al. 1999; Derichs \& Rauch 2000; KrüGer 2003). Der Ansatz greift auf Verwundbarkeitskonzepte zurück 
und hat zum Ziel, den gesamten Vulnerabilitätskontext von Haushalten zu erfassen. Die als livelihood bezeichnete Lebenshaltung umfasst alle Fähigkeiten, Ausstattungen und Handlungen, die zur alltäglichen Existenzsicherung erforderlich sind. Im Mittelpunkt der Analyse dieses Lebenshaltungssystems stehen zunächst fünf Ressourcenbündel (assets): Humankapital (z.B. Wissen, Fähigkeiten, Fertigkeiten, Gesundheit), Naturkapital (wie Land, Wasser, Böden), Sozialkapital (Einbindung in soziale Netzwerke, gesellschaftlicher Status), Sachkapital (u.a. infrastrukturelle Ausstattung, Produktionsmittel) und Finanzkapital (wie Einkommen, Kreditzugang). Untersucht wird nun die Bedeutung dieser Ressourcenbündel für die jeweilige Lebensabsicherung eines Haushalts bzw. einer sozialen Gruppe. Welchen Stellenwert nimmt eine bestimmte Ressource im System der Existenzsicherung ein? Sind notwendige Ressourcen überhaupt vorhanden und sind sie frei zugänglich? In welchen externen politisch-ökonomischen und institutionellen Rahmen sind die Verfügbarkeit dieser Ressourcenbündel und der Zugang zu ihnen eingebettet? Schließlich geht es um die Analyse der Lebenshaltungsstrategien, die die sozialen Akteure entwickeln und einsetzen, um ihre Ressourcenausstattung zu verbessern und ihre Verwundbarkeit auf einem möglichst niedrigen Niveau zu halten.

Der livelihood-Ansatz basiert auf der Überlegung, dass die materiellen und immateriellen Vermögenswerte, Fähigkeiten und Kapazitäten eine zentrale Rolle bei der Abpufferung und Bewältigung von Stress-Situationen, Krisen und Katastrophen spielen. Gelingt es den betreffenden Haushalten, alle notwendigen Ressourcen zu erschließen und zu mobilisieren, dann betreiben sie eine wirksame Sicherung ihrer Lebenshaltung. Existenzsicherungsstrategien sind also nichts anderes als der - mehr oder weniger erfolgreiche - Versuch der Mobilisierung der obgenannten Kapitalbündel. Gesellschaftliche Konflikte entstehen nun, wenn es zu Konkurrenzkämpfen um knappe Ressourcen kommt oder institutionelle Regelungen einzelne Bevölkerungsgruppen in eine Situation der Benachteiligung rücken, aus der sie sich zu befreien versuchen (müssen). Unter Umständen führt auch das Fehlen etablierter Regelungen, das sich z.B. als institutionelle Lücke in Phasen ausgeprägter gesellschaftlicher Transformation manifestiert, zu Konflikten. Wird die Mobilisierung der Ressourcenbündel verhindert und sind autonome Sicherungsmechanismen nicht mehr alleine wirksam, so kommt es zur Krise. Sie konstituiert einen Wendepunkt, ab dem externe Interventionen notwendig werden, um das Lebenshaltungssystem vor dem Zusammenbruch zu bewahren. Auslöser dieser Krise können Störungsereignisse sein, die als Gefahrenmomente Menschen oder deren Vermögens- und Sachwerte beeinträchtigen. Kollabiert das System der
Lebenshaltung, weil erfolglos oder überhaupt nicht interveniert wurde, ist das Stadium der Katastrophe erreicht. Sie steht damit am Ende eines sich zunehmend destabilisierenden Handlungs- und Kommunikationsprozesses.

Das livelihood-Modell kennt also zwei Ursachen für Konflikte, Krisen und Katastrophen:

1. das Nicht-Vorhandensein von für die Lebenshaltung ausreichenden Ressourcenbündeln und Kapitalausstattungen, und/oder

2. das Nicht-Funktionieren von Sicherungsstrategien der betroffenen Menschen.

Damit identifiziert das Modell wichtige Ansatzpunkte für Projekte der Entwicklungszusammenarbeit, die darauf abzielen, die Überlebenssicherung verwundbarer Gruppen zu stärken. Trotz seines multisektoralen Charakters und der Berücksichtigung der Handlungspielräume Betroffener greift das Konzept aber noch immer zu kurz. Die Rahmenbedingungen für das Handeln, für die Anpassungsstrategien und Bewältigungskapazitäten sind zwar angesprochen, aber befriedigende Erklärungen für die Beweggründe dieses Handelns werden kaum geliefert. Verborgen bleiben auch die eigentlichen Ursachen des Scheiterns bestimmter Handlungsrationalitäten. Aus dem Modell lassen sich ferner die Risikobedingungen, unter denen Alltagshandeln und Existenzsicherung stattfinden, nur indirekt ableiten. Risiken werden bestenfalls diffus als gesellschaftlich interpretierte und institutionell konditionierte Gefahren beschrieben, die im Zusammenhang mit Bewältigungsmechanismen auftreten. Verwundbarkeit und das Risiko einer Existenzkrise sind niedrig, wenn eine Gesellschaft langfristige und nachhaltige Präventionsmaßnahmen entwickelt hat und die Notwendigkeit zu unvorbereiteter, rascher Reaktion auf Gefahrenereignisse gering ist. Am verwundbarsten sind Bevölkerungsgruppen, denen kein Präventionsinstrumentarium zur Verfügung steht und die spontan, kurzfristig, unkoordiniert und reaktiv handeln müssen (Abbildung 1). Aufgrund dieser Defizite des livelihood-Konzepts erscheint es sinnvoll, dem Modell weitere Ansätze zur Seite zu stellen, die lokales Handeln im Kontext von Krisen und Katastrophen begreiflicher machen.

\section{Grundkonzeptionen der Risikosoziologie}

Das livelihood-Modell sowie der damit verbundene Begriff der Verwundbarkeit finden in der Soziologie wenig Beachtung. Und dies, obwohl sie sowohl strukturelle als auch handlungsrelevante Aspekte aufgreifen. Die von ihnen angesprochenen Sachverhalte finden sich aber in der Auseinandersetzung mit dem Begriff des Risikos wieder. Angeregt wurde die Diskussion von UlRICH BeCK, der in seinem Buch über die Risiko- 


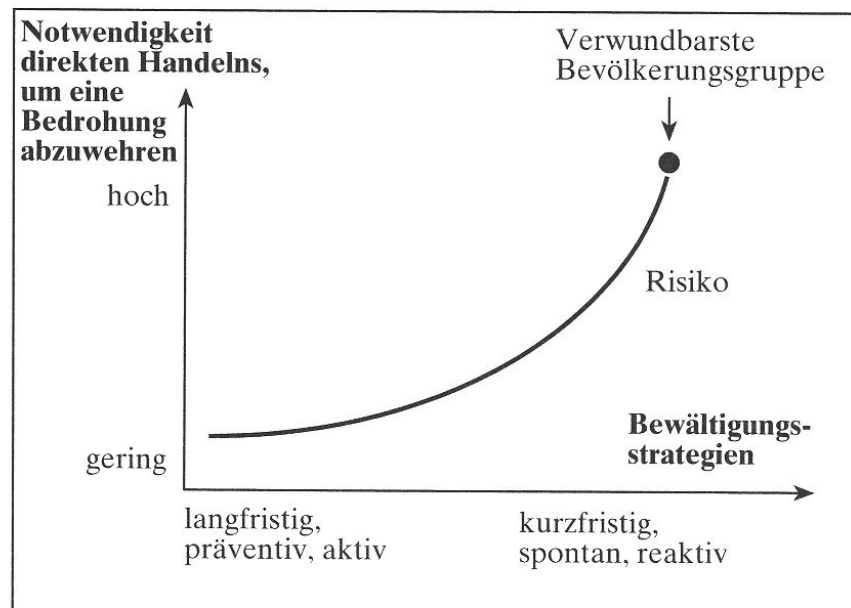

Abb. 1: Risiko, Bewältigung und Verwundbarkeit Erläuterung: Die Verwundbarkeit einer Bevölkerungsgruppe nimmt entlang der "Risiko-Linie» von links unten nach rechts oben zu. Beispiel: Eine Gemeinschaft, der ein erprobtes Präventivinstrumentarium zur Abwehr von Bedrohungen zur Verfügung steht (auf der $x$-Achse weit links) und die gleichzeitig nur wenig Anlass hat, dieses Bewältigungsinstrumentarium einsetzen zu müssen (weil sie z.B. nur selten akuten Gefahren ausgesetzt ist; auf der y-Achse weit unten), besitzt ein geringes Gefährdungsrisiko und ist wenig verwundbar. Eine Gemeinschaft jedoch, die kaum Bewältigungsmöglichkeiten besitzt (auf der $x$-Achse weit rechts), aber häufig auf unmittelbare Gefahren reagieren muss (auf der $y$-Achse ganz oben), ist stark verwundbar.

Risk, vulnerability and coping strategies

Risque, maîtrise et vulnérabilité

Entwurf: F. KrÜGER, E. MACAMO

gesellschaft (1986) ein neues Forschungsfeld eröffnete, das der Soziologie neue Zugänge zum Umgang mit Extremsituationen gewährte. Entscheidend für die erfolgreiche Einführung des Begriffs Risiko war neben dem Unbehagen mit der technologischen Gesellschaft ein allgemeines Bedürfnis nach der Klärung der alten Frage, was moderne Gesellschaften zusammenhält.

In den 1970er-Jahren setzte sich in der Soziologie eine Betrachtungsweise durch, die der Frage nachging, wie Gesellschaften dazu beitragen, Sicherheit herzustellen. Federführend waren im deutschsprachigen Raum die Arbeiten von Kaufmann (1973) und Nowotny (1989). Während Kaufmann in seinen Ausführungen das Sozialsystem mit seinem Versicherungsprinzip in den Vordergrund stellte, beschäftigte sich Nowotny mit der Frage, wie die rationale Aneignung der Natur durch die Wissenschaft bezeichnend für den modernen Umgang mit Unsicherheit wurde.

Eine wichtige Errungenschaft der Konzentration auf die Sozialpolitik und Wissenschaft war die sozialwissenschaftliche Erkenntnis, wonach nicht die Gefahrenereignisse an sich von Bedeutung sind, sondern der gesellschaftliche Umgang damit. In einem drei-stufigen Modell versuchte man, diesen Umgang mit Unsicherheit über die Begriffe Risiko, Krise und Katastrophe zu thematisieren. Zunächst verstand man unter «Risiko» die gesellschaftliche Wahrnehmung einer externen Bedrohung; «Krise» bezog sich auf die Realisierung einer Bedrohung in der Gesellschaft und die Auseinandersetzung um die bessere Lösung; mit dem Begriff der «Katastrophe» meinte man den Verlust der gesellschaftlichen Fähigkeit, die in der Krise konkret gewordene Bedrohung abzuwenden. Da man von der Annahme ausging, dass es der modernen Gesellschaft weitgehend gelungen sei, Katastrophen zu verhindern, konzentrierte sich die Aufmerksamkeit auf die ersten Stufen des Modells. Wie werden Risiken in der Gesellschaft wahrgenommen und wie setzt sie sich damit auseinander?

Diese Fragestellung hat zu drei Ansätzen geführt, die von DeBorah Lupton (1999: 35) als (a) realist epistemological position, (b) weak constructionist epistemological position und (c) strong constructionist epistemological position definiert wurden. Es handelt sich um erkenntnistheoretische Ansätze, die sich in der Art und Qualität der Frage nach der Beschaffenheit von Risiko und der gesellschaftlichen Auseinandersetzung damit unterscheiden. Der stark konstruktivistische Ansatz wird in Deutschland kaum verfolgt. Für unsere Überlegungen reicht es, wenn wir uns auf den realistischen und den schwach konstruktivistischen Ansatz konzentrieren.

Realistische Ansätze gehen davon aus, dass Risiken objektive Gegebenheiten sind, die es in der Natur gibt. Sie stellen Bedrohungen und Gefahren dar, die genau beobachtet und gemessen werden können. Dabei spielt der gesellschaftliche Umgang damit keine große Rolle. Es gibt eine sehr umfangreiche Literatur zu diesem Sachverhalt, die sich in den 1980er- und 90erJahren vor allem im Begriff «Risikoabschätzung» niederschlug. Diese Literatur bediente sich zum einen wirtschaftswissenschaftlicher Überlegungen zum Risiko (KNIGHT 1965) und zum anderen Bemühungen aus naturwissenschaftlichen Kreisen, die negativen Folgen wissenschaftlicher Produkte unter Kontrolle zu bringen. Risikoabschätzung entwickelte sich $\mathrm{zu}$ einer regelrechten Wissenschaft, die für unser Vorhaben deshalb interessant ist, weil sie von der positivistischen Überzeugung geprägt war, dass Extremsituationen ohne Rücksicht auf gesellschaftliche Prozesse und nur durch den überlegenen Einsatz technischer Mittel abgewendet werden könnten.

Im Gegensatz zu dieser realistischen Perspektive gehen 
die konstruktivistischen Ansätze nicht davon aus, dass Risiken objektiven Tatsachen entsprechen. Vielmehr verstehen sie sie als Ergebnis davon, wie Gesellschaften externe Bedrohungen wahrnehmen. Risiko wird in den beiden konstruktivistischen Ansätzen als Konstrukt betrachtet. Das Augenmerk richtet sich auf das Gesellschaftsverständnis von Gefahr und auf die Mechanismen, die Menschen zur Bewältigung dieser Gefahren entwickeln. Der schwach konstruktivistische Ansatz akzeptiert die Existenz von objektiven Gefahren. Er besteht jedoch darauf, dass das von diesen Gefahren dargestellte Risiko erst in der gesellschaftlichen Auseinandersetzung sichtbar wird. Mit anderen Worten argumentiert der schwach konstruktivistische Ansatz, dass Gefahren nicht losgelöst von sozialen und kulturellen Prozessen zu betrachten sind. Es sind diese sozialen und kulturellen Prozesse, die Gefahren vergegenständlichen, d.h. sie in der öffentlichen Wahrnehmung als Risiken darstellen.

ULRICH BECKS «Risikogesellschaft» spielt auf diesen Sachverhalt an. Er vertritt die Ansicht, dass die Gefahren eines Atomunfalls oder einer Lebensmittelvergiftung objektiv vorhanden seien, aber noch kein Risiko darstellen. Erst durch das Misstrauen der Bevölkerung der Atomwirtschaft oder der Lebensmittelindustrie gegenüber, das sich z.B. in Bürgerinitiativen u.ä. niederschlägt, werden diese Gefahren als Risiken vergegenständlicht. Der Begriff Risikogesellschaft bezieht sich nicht auf eine Gesellschaft, die von Gefahren bedroht ist, sondern auf eine Gesellschaft, die solche Gefahren thematisiert. GIDDENs führt diese Gedanken mit seiner Feststellung weiter, dass moderne Gesellschaften deshalb als Risikogesellschaften zu verstehen seien, weil sie die Fähigkeit besitzen, sich selbst in Frage zu stellen. Risiken, so das Argument, sind Ergebnis der reflexiven Moderne (Beck, Giddens \& LASH 1994).

Die enge Kopplung von Risikowahrnehmung und Moderne hat einige Autoren dazu bewogen, den Begriff «Risiko» nur auf moderne Gesellschaften zu beschränken, wobei darunter der Entwicklungsstand verstanden wird. WolfGang Bonss (1995) beispielsweise spricht den auch von Mary Douglas untersuchten Lele die Fähigkeit ab, Risiken zu erkennen. Als Grund dafür führt er das mangelnde technische Wissen der Lele an, das ihnen nicht erlaube, Gefahren objektiv zu erkennen. Douglas bedient sich des gleichen Ansatzes, kommt jedoch zu anderen Ergebnissen. Ihr geht es darum, wie in bestimmten soziokulturellen Kontexten bestimmte Gefahren als Risiken verstanden werden und andere nicht. Sie argumentiert, dass Risiken stets Übersetzungsprozessen unterliegen, anhand derer Gesellschaften Gefahren als Bedrohung thematisieren (Douglas \& WILDAVSKy 1982; Douglas 1992).
In ihrer konstruktivistischen Prägung versteht die Risikosoziologie Risiken also nicht als objektive externe Bedrohungen, sondern als vergegenständlichte Gefahren, die als solche in der gesellschaftlichen Auseinandersetzung entstanden sind. Die von NiKLas LuHMANN (1991) unternommene Begriffsklärung macht dies deutlich. LuHMANN unterscheidet prinzipiell zwischen Gefahr und Risiko. Seiner Ansicht nach besteht dieser Unterschied in der Zurechenbarkeit von Ereignissen. Gefahren sind Ereignisse, die auf die Umwelt zurückzuführen sind. Risiken dagegen sind Ereignisse, die dem sozialen Handeln zuzurechnen sind. Ein Risiko stellt also ein gesellschaftliches Kalkül dar. Die Gesellschaft nimmt bewusst eine Gefahr in Kauf, d.h. sie rechnet dem eigenen Handeln mögliche negative Folgen zu. Somit besteht der Unterschied zwischen Gefahr und Risiko in der Möglichkeit, Gefahren durch Eingriffe in die Natur in Risiken zu übersetzen. Risiko ist für LuHMANN kein negativ besetzter Begriff, sondern die Bedingung der Möglichkeit für soziales Handeln. Damit ist ein zweiter Gesichtspunkt angesprochen: Gesellschaften konstituieren sich anhand der Erzeugung von Risiken, d.h. das Abwenden von Gefahren impliziert die Produktion von Risiken. Was sich zunächst widersprüchlich anhört, lässt sich handlungstheoretisch erklären. Aus der Sicht der verstehenden Soziologie bestehen soziale Ordnungen aus dem sozialen Handeln. Aufgrund dieser konstitutiven Rolle orientiert sich das soziale Handeln darauf, zuverlässige und stabile Rahmen für handelnde Subjekte zu schaffen (Weber 1972). Die auf diese Weise hergestellte Zuverlässigkeit und Stabilität beschreiben das Maß an Berechenbarkeit, die für den normalen Alltag notwendig ist. Risikokalkulationen sind Formen des Umgangs mit Unsicherheit, anhand derer Gesellschaften Rahmen für berechenbares soziales Handeln schaffen. Im Prozess der Übersetzung von Gefahren in Risiken wird anhand der Handlungsbezogenheit, Entscheidungsbezogenheit und Verantwortbarkeit von gesellschaftlichen Abhandlungen Berechenbarkeit im Alltag hergestellt.

Was existenzgefährdend ist, wird also in der Gesellschaft bestimmt. In einer Studie über die lokale Interpretation einer Überschwemmung, die sich im Jahre 2002 im Süden Mosambiks ereignete, wurde beispielsweise festgestellt, dass nicht die Überschwemmung an sich als Katastrophe wahrgenommen wird, sondern die Zeit danach (Масамо 2003). Die Not- und Katastrophenhilfe konzentrierte ihre Aktionen auf die Zeit der tatsächlichen Überschwemmung und brachte damit ihre Überzeugung zum Ausdruck, dass sie die Katastrophe in eben dieser Überschwemmung sah. Die lokale Bevölkerung jedoch bewertete die Überschwemmung als normales Ereignis ( Hochwasser ist ein Gast, der kommt, drei Tage bleibt und dann weiter zieht»), das dem Land und der Gemeinschaft «gut tue». 
Wasser reinigt den Boden, tötet das Ungeziefer und macht das Land fruchtbar. Einige Menschen kommen dabei ums Leben, manche verlieren ihr Hab und Gut. Solange jedoch die Gemeinschaft ihre erprobten Bewältigungsstrategien einsetzen kann, ist eine Überschwemmung an sich keine Katastrophe. Soweit die Gemeinschaft nach dem Abzug des Wassers die Felder wieder ohne fremde Unterstützung, sondern mit eigenen bewährten Mitteln bestellen kann, bleibt das Hochwasser ein normales Ereignis. Was die Überschwemmung zu einer Katastrophe machte, so die lokale Interpretation, war der unerwartete Eintritt einer Dürre danach. Die Betroffenen konnten daher die Überschwemmung nicht mehr als Routineereignis in die Normalität ihrer lebensweltlichen Erfahrung einordnen.

\section{Fazit: Ganzheitliche, handlungsorientierte Analyse von Existenzunsicherheiten}

Wenn Risiken gesellschaftliche Aneignungen von Gefahren mit dem Ziel der Herstellung von Berechenbarkeit darstellen, so entstehen existenzgefährdende Krisen dann, wenn eine Gemeinschaft grundlegende Unsicherheiten zeigt, auf welche Art und Weise die Aneignung von Gefahren zu erfolgen hat. Die betroffene Gesellschaft gerät förmlich ins Schlingern und Trudeln, ein angemessener Kurs der Gefahrenbewältigung kann kaum noch verfolgt werden. Zur Katastrophe kommt es, wenn diese Unsicherheiten so eklatant werden, dass adäquate Mittel der Gefahrenaneignung nicht mehr gefunden werden können (Abbildung 2).

Die in den livelihood-Modellen betonten Bewältigungsstrategien der Lebensabsicherung stellen somit letztlich nichts anderes dar als den «Schlinger- und Trimmkurs» der Gefahrenaneignung (vgl. DOMBROwSKY 1996: 71). Es liegt also nahe, den Untersuchungsansatz des livelihood-Modells zu ergänzen und die gesellschaftliche Erzeugung von Risiken bei der Analyse von Existenzsicherung ebenfalls zu berücksichtigen. Die Erzeugung von Risiken findet im Prozess der Übersetzung von Gefahren in Risiken statt. Dieser Prozess der Übersetzung beschreibt eine Entscheidungsstruktur, die aus drei Elementen besteht: (a) Handlungsbezogenheit, (b) Entscheidungsbezogenheit und (c) Verantwortbarkeit. Diese Elemente schlagen eine Brücke zwischen Gefahr auf der einen Seite und Risiko auf der anderen. Die Übersetzung der Gefahr einer Überschwemmung in ein Risiko impliziert zunächst entsprechendes soziales Handeln: z.B. Meidung der Flussaue als Wohnstandort, Entwicklung von Anbautechniken, die Hochwässer als natürliche Bewässerung nutzen. Lokale Gemeinschaften haben für die Chiffrierung von Gefahren in Risiken Handlungsreper-

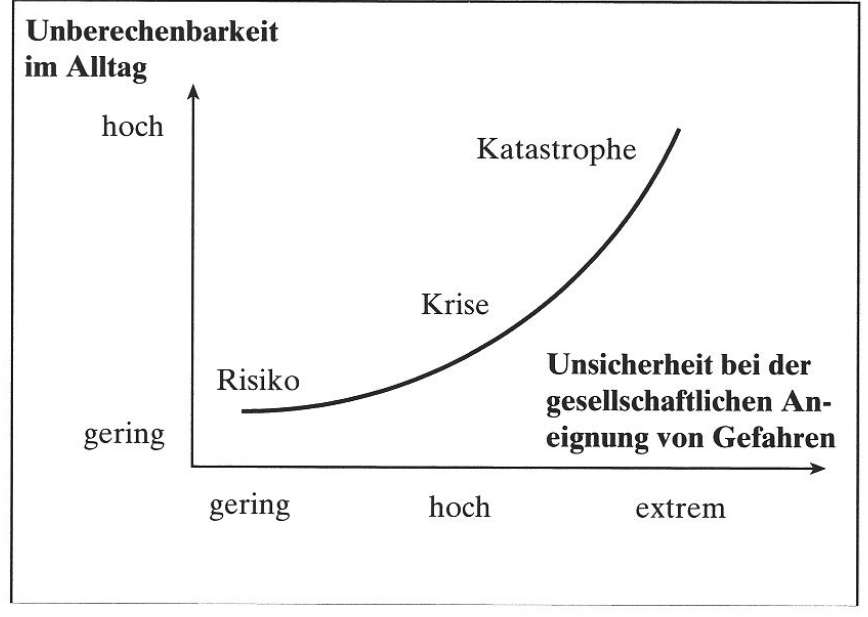

Abb. 2: Existenzgefährdung aus risikosoziologischer Sicht

Livelihood security in the context of sociology of risk

Menace des conditions d'existence dans une optique sociologique du risque

Entwurf: F. KrüGER, E. MacAmo

toires, die sowohl ihre Wahrnehmung der Bedrohung als auch ihre bewährten Bewältigungsstrategien zum Ausdruck bringen. Zur Abschätzung der Krisenbewältigungsfähigkeit einer lokalen Gemeinschaft gilt es zunächst, deren Handlungsrepertoire zu erfassen. Dann muss der analytischen Frage nachgegangen werden, welche Restgefahren von der Gemeinschaft bewusst in Kauf genommen werden, denn diese markieren das subjektive Sicherheitsgefühl. Als nächstes muss die Entscheidungsbezogenheit beschrieben werden: Wer trifft welche Entscheidungen wann in Bezug auf Bewältigungsmaßnahmen? Sind die Entscheidungsinstanzen in der lokalen Wahrnehmung kompetent genug? Welche Kriterien werden zur Beurteilung der Kompetenz lokal zugrunde gelegt? Die Entscheidungsbezogenheit spielt eine wichtige Rolle bei der Herstellung von Berechenbarkeit. Von ihr hängt die Frage ab, ob man sich auf ein Handeln verlassen kann oder nicht. Das dritte Element ist die Verantwortbarkeit. Wer trägt die Verantwortung für die Präventions-, Anpassungs-, Abwehr- und Bewältigungsmaßnahmen? Wer erfüllt welche Aufgaben und an wen wendet man sich, wenn Schwierigkeiten auftreten? Das Element der Verantwortbarkeit beschreibt zum einen die Arbeitsteilung in einer Gefahr/Risiko-Situation und gibt der Gemeinschaft zum anderen Anhaltspunkte für die Zurechnung von negativen und positiven Folgen. In der Terminologie des livelihood-Modells sind Entscheidungsinstanzen und Problemlösungsautoritäten vor allem im Bereich des Human- und Sozialkapitals einer Gesellschaft zu verorten. 
Wichtig bei der Analyse des Übersetzungsprozesses ist die Erkenntnis, dass tatsächliche Gefahren nur die eine Seite der Medaille sind. Im Grunde werden alle externen Faktoren analytisch als Gefahren betrachtet, die von der lokalen Gemeinschaft in Risiken übersetzt werden müssen. Solange dieser Übersetzungsprozess nicht oder nicht erfolgreich stattfindet, stellen externe Interventionen (beispielsweise Hilfslieferungen) keine Existenzsicherungsinstrumente dar, sondern Bedrohungen für das lokale soziale Handeln und für den entsprechenden Bezugsrahmen. Diese Erkenntnis zielt darauf ab, entwicklungspolitische Maßnahmen nicht nur als positive Faktoren in die Analyse einzubeziehen, sondern sie zu thematisieren. Eine von DombrowSKI (1996) an die internationalen Katastrophenhilfsdienste gerichtete Kritik gibt zu bedenken, dass nicht nur Naturereignisse problematisch sind, sondern auch die zu ihrer Bewältigung eingesetzten Mittel. Sie entsprechen nämlich oft Übersetzungsprozessen, die in anderen gesellschaftlichen Kontexten stattgefunden haben, und damit einer bestimmten Sicht von Katastrophe, die von den Betroffenen vor Ort eventuell gar nicht geteilt wird. Aus diesem Grunde ist es wichtig, bei der Beschreibung und Analyse der Erzeugung von Risiken auch Lösungen und deren Einfluss auf lokale Kontexte zu thematisieren.

Mit der Kombination verwundbarkeitsbezogener und risikosoziologischer Analyseansätze lässt sich eine ganzheitlichere Betrachtung von Lebenssituationen unter Krisen- oder Katastrophenbedingungen erzielen. Die handlungstheoretische Perspektive sollte dabei besonders deutlich betont werden. Die Relevanz und die Nützlichkeit einer auf livelihood-Ansätzen bezogenen Beschreibung von Lebenssituationen kommen nämlich erst in der Einbeziehung des lokalen Handelns wirklich zum Tragen. Das soziale Handeln unter Krisen- oder Katastrophenbedingungen zielt zunächst auf die Herstellung von Berechenbarkeit im Alltagshandeln - dem trägt die Kombination beider Ansätze Rechnung. Freilich muss in der Forschungspraxis bedacht werden, dass beim Einbringen beider Konzeptionen die Anforderungen an die Expertise der Forschenden und der Umfang der Methodensets so groß werden, dass sie von Einzelnen eventuell gar nicht mehr bewältigt werden können. Sowohl in der Feldforschung als auch in der Entwicklungspraxis ist interdisziplinäres Arbeiten im Team Voraussetzung zur erfolgreichen Operationalisierung der hier vorgestellten Analyseansätze.

\section{Literatur}

BECK, U. (1986): Risikogesellschaft. Auf dem Weg in eine andere Moderne. - Frankfurt: Suhrkamp.

Beck, U., Giddens, A. \& S. LAsh (1994): Reflexive Modernization - Politics, Tradition and Aesthetics in the Modern Social Order. - Cambridge: Polity Press. Blaikie, P., Cannon, T., Davis, I. \& B. Wisner (1994): At Risk. - London, New York: Routledge.

Bonss, W. (1995): Vom Risiko - Unsicherheit und Ungewissheit in der Moderne. - Hamburg: Hamburger Edition.

CARney, D. et al. (1999): Livelihoods Approaches Compared. - London: Department for International Development (DFID).

DERICHS, A. \& T. RAUCH (2000): LRE und der «Sustainable Rural Livelihoods»-Ansatz. - In: Entwicklungsethnologie 9, Nr. 2: 60-78.

Dombrowsky, W.R. (1996): Falsche Begriffe, falsches Begreifen, schädliches Zugreifen vor Ort: Über die Folgen verkehrten Denkens beim Gutgemeinten. - In: Hanisch, R. \& P. Mossmann (Hrsg.): Katastrophen und ihre Bewältigung in den Ländern des Südens. - = Schriften des Deutschen Überseeinstituts 33: 61-72.

Douglas, M. (1986): Risk Acceptability According to the Social Sciences. - London: Routledge \& Kegan Paul.

Douglas, M. (1992): Risk and Danger. - In: DougLAS, M.: Risk and Blame. Essays in cultural theory. London, New York: Routledge: 38-54.

Douglas, M. \& A. Wildavsky (1982): Risk and Culture. - Berkeley, Los Angeles, London: University of California Press.

HewitT, K. (1997): Regions at Risk. A geographical introduction to disasters. - Harlow: Longman.

Johnston, R., Gregory, D., Pratt, G. \& M. Watts (Hrsg.) (2000): The Dictionary of Human Geography; (Eintrag «risk»). - 4. Auflage, Oxford: Blackwell.

KaufMann, F.-X. (1973): Sicherheit als soziologisches und sozialpolitisches Problem. Untersuchungen zu einer Wertidee hochdifferenzierter Gesellschaften. Stuttgart: Enke.

Knight, F. (1965): Risk, Uncertainty and Profit. - Chicago: University of Chicago Press.

KRÜGER,F. (2003): Handlungsorientierte Entwicklungsforschung: Trends, Perspektiven, Defizite. - In: Petermanns Geographische Mitteilungen 147, 1: 6-15.

Luhmann, N. (1991): Soziologie des Risikos. - Berlin: De Gruyter.

Lupton, D. (1999): Risk. - London: Routledge.

Macamo, E. (2003): Nach der Katastrophe ist die Katastrophe - die 2000er Überschwemmung in der lokalen Wahrnehmung in Mosambik. - In: Clausen, L., Geenen, E. \& E. Macamo (Hrsg.): Entsetzliche soziale Prozesse. Theoretische und empirische Annährungen. - Hamburg: Lit Verlag (im Druck).

NowotNy, H. (1989): Sicherheit und Komplexität: über den Umgang mit Unsicherheit. - In: Zeitschrift für Wissenschaftsforschung 5: 3-12.

PoHL, J. (1998): Die Wahrnehmung von Naturrisiken in der «Risikogesellschaft». - In: HeINRITZ, G., WiESSNER, R. \& M. Winiger (Hrsg.): Verhandlungsband des 51. Deutschen Geographentags Bonn 1997, Bd. 2, Stuttgart: 153-163. 
SPITTLER, G. (1994): Hungerkrisen im Sahel. - In: Geographische Rundschau 46, 7-8: 408- 413.

SPITTLER, G. (1996): Nomaden in Dürren und Hungerkrisen - Plädoyer für eine neue Perspektive. - In: Hanisch, R. \& P. Mossmann (Hrsg.): Katastrophen und ihre Bewältigung in den Ländern des Südens. - = Schriften des Deutschen Überseeinstituts 33: 78-95.

Wissenschaftlicher Beirat DER BUndesRegierung Globale UmweltVeränderungen (WBGU) (1999): Welt im Wandel: Strategien zur Bewältigung globaler Umweltrisiken - Zusammenfassung. - Bremerhaven: Wissenschaftlicher Beirat der Bundesregierung Globale Umweltveränderungen.

Weber, M. (1972): Wirtschaft und Gesellschaft. - 5. Auflage, Tübingen: J.C.B. Mohr (Paul Siebeck).

YATES, F. (Hrsg.)(1992): Risk-taking Behavior. - Chichester: John Wiley \& Sons.

\section{Zusammenfassung: Existenzsicherung unter Risiko- bedingungen - Sozialwissenschaftliche Analyse- ansätze zum Umgang mit Krisen, Konflikten und Katastrophen}

In der Entwicklungsforschung spielen für die Analyse von Alltagshandeln und Existenzsicherung Risikokonzeptionen eine wichtige Rolle. Der Risikobegriff selbst bleibt dabei jedoch häufig diffus und ambivalent. Verwundbarkeits- und livelihood-Modelle werten Krisen und Katastrophen als Ergebnisse gesellschaftlicher Prozesse, die zum Zusammenbruch lebensnotwendiger Ressourcen oder zum Versagen adäquater Bewältigungsstrategien führen. Die Risikobedingungen des Alltagshandelns werden zwar angesprochen, aber befriedigende Erklärungen für die Beweggründe dieses Handelns werden kaum geliefert. Der Beitrag zeigt, dass Ansätze der Risikosoziologie die geographischen Konzepte hier sinnvoll ergänzen können. Aus Sicht der Risikosoziologie ist Existenzgefährdung aus dem gesellschaftlichen Umgang mit Unsicherheit zu verstehen. Dieser Umgang zeichnet sich durch das dem sozialen Handeln zugrundeliegende Streben nach Herstellung von Berechenbarkeit im Alltag aus. Berechenbarkeit entsteht als Folge eines Kommunikationsund Handlungsprozesses, in dem Betroffene Gefahren in Risiken übersetzen. Aus der Kombination von livelihood- und Risiko-Konzepten ergeben sich daher nicht nur wertvolle Hinweise zur entwicklungstheoretischen Erklärung von Existenzgefährdung, sondern auch vielversprechende Ansatzpunkte für die entwicklungspraktische Arbeit.

\section{Summary: Safeguarding Livelihoods under Condi- tions of Risk - Social scientific analytical approaches to coping with crises, conflicts and disasters}

In the context of development research, notions of risk play an important role in the analysis of action in everyday-life and livelihood security. Often, however, the notion of risk itself remains ambiguous and vague. Vulnerability and livelihood models assess crises and disasters as the result of societal processes which lead either to the collapse of resources which are vitally important or to the failure of adequate coping strategies. While conditions of risk are dealt with in the context of action in everyday-life no satisfactory explanations for the underlying reasons for these actions are provided. This paper seeks to show that approaches from the sociology of risk may complement geographical notions in a meaningful way. From the perspective of sociology, livelihood risks should be understood from a viewpoint which focuses on the way societies come to terms with insecurity. This is characterised by the pursuit of different ways of securing calculability in everyday-life which is typical of social action. Calculability is a result of communicative and action processes in which those affected by hazards translate them into risks. The combination of both livelihood approaches and risk notions produces not only important insights to livelihood security in the context of development theory, but also forms a promising starting-point for practical development work.

\section{Résumé: La sécurisation de l'existence dans un con- texte de risque - approches analytiques en sciences sociales sur le comportement face aux crises, conflits et catastrophes}

Dans le domaine de la recherche sur les questions de développement, le concept de risque joue un rôle important pour l'analyse de l'action quotidienne et des problèmes liés à la sécurisation de l'existence. La notion de risque elle-même demeure toutefois ambivalente et diffuse. Les modèles livelihood et de vulnérabilité considèrent les crises et les catastrophes comme des résultats de processus sociaux qui ont conduit à l'effondrement de ressources indispensables à l'existence ou à la faillite de stratégies de maîtrise adéquates. Même si l'on parle des conditions de risque de l'action quotidienne, on a du mal à apporter des réponses satisfaisantes pour les motivations qui sont à la base de cette action. Cette contribution montre que les approches de la sociologie du risque peuvent être un complément non négligeable aux concepts développés par les géographes. Dans la perspective géographique, on ne peut comprendre le risque pour la vie qu'à partir de la manière dont la société fait face à l'insécurité. Ce processus se caractérise par l'introduction de la calculabilité dans le quotidien, celle-ci étant à la base de l'action sociale. La calculabilité elle-même résulte d'un processus de communication et d'action dans lequel les victimes traduisent les dangers en risques. À partir de la combinaison entre les notions de livelihood et de risque on peut retrouver non seulement de précieux indices qui permettent d'expliquer une menace pour l'existence dans la perspective d'une théorie du développement, mais aussi un point de 
départ très prometteur pour le travail pratique sur les problèmes du développement.

\section{Didaktische Hinweise}

- In welchem Verhältnis stehen die Begriffe Gefahr Risiko - Verwundbarkeit - Krise - Katastrophe zueinander? Wie lassen sie sich sinnvoll unterscheiden?

- Was untersucht die livelihood-Forschung und wo liegen deren Grenzen? Was sind aus Sicht der livelihood- und Verwundbarkeitsmodelle die wichtigsten Ursachen für gesellschaftliche Krisen, Konflikte und Katastrophen?

- Warum können Risiko-Konzepte eine wertvolle Grundlage für die Analyse von Existenzsicherung und Alltagshandeln in Entwicklungsländern bilden?

- Welchen Stellenwert erfährt das Risiko vor dem Hintergrund des realistischen und des konstruktivistischen Ansatzes? Unter welchen Voraussetzungen verändern sich Gefahren zu abwägbaren Risiken?
- Was sind aus Sicht der livelihood- und Verwundbarkeitsmodelle die wichtigsten Ursachen für gesellschaftliche Krisen, Konflikte und Katastrophen?

Prof. Dr. Fred Krïger, Institut für Geographie der Universität Erlangen-Nürnberg, Kochstr. 4, D-91054 Erlangen.

e-mail: fkrueger@geographie.uni-erlangen.de

Dr. Elísio Macamo, Entwicklungssoziologie, Universität Bayreuth, D-95440 Bayreuth.

e-mail:Elisio.Macamo@uni-bayreuth.de

Manuskripteingang/received/manuscrit entré le 18.11.2002

Annahme zum Druck/accepted for publication/accepté pour l'impression: 8.4 .2003 